\title{
Polymerization enabled reduction of the electrically induced birefringence change in nematic liquid erystals
}

\author{
Jiahao Chen (陈佳浩) $)^{1, \dagger}$ ，Chaoyi Li (李超逸 $)^{2,3, \dagger}$ ， Luyao Sun (孙路瑶) ${ }^{1}$ ， Lingling Ma (马玲玲 $)^{2,3}$ ， Bingxiang Li (李炳祥) $)^{1,2,4^{*}}$ ， \\ and Yanqing Lu (陆延青) $)^{2,3^{* *}}$ \\ ${ }^{1}$ College of Electronic and Optical Engineering and College of Microelectronics, Nanjing University of Posts and Telecommunications, \\ Nanjing 210023, China \\ ${ }^{2}$ National Laboratory of Solid State Microstructures, Nanjing University, Nanjing 210093, China \\ ${ }^{3}$ Collaborative Innovation Center of Advanced Microstructures \& College of Engineering and Applied Sciences, Nanjing University, Nanjing 210093, China \\ ${ }^{4}$ Advanced Materials and Liquid Crystal Institute, Kent State University, Kent, OH 44242, USA
}

*Corresponding author: bxli@njupt.edu.cn

${ }^{*}$ Corresponding author: yqlu@nju.edu.cn

Received October 17, 2021 | Accepted December 2, 2021 | Posted Online January 4, 2022

\begin{abstract}
Electric fields modify the optical properties of nematic liquid crystals (NLCs) by changing the nematic molecular orientation or order parameters, which enables electro-optic applications of NLCs. However, the field-induced optic change is undesirable in some cases. Here, we experimentally demonstrate that polymer stabilization weakens the birefringence change of NLCs caused by the nanosecond electrically modified order parameter effect. The birefringence change is reduced by $65 \%$ in the NLC doped with $25 \%$ reactive monomer, which is polymerized close to the nematic-to-isotropic phase transition. This technique could be used in liquid crystal devices where the birefringence change is unfavored.
\end{abstract}

Keywords: liquid crystal; electro-optical response; birefringence; polymerization.

DOI: 10.3788/COL202220.023201

\section{Introduction}

Nematic liquid crystals (NLCs) have a wide range of electrooptic applications ${ }^{[1-14]}$, such as displays ${ }^{[1-3]}$, optical shutters ${ }^{[4,5]}$, modulators $^{[6,7]}$, splitters ${ }^{[8,9]}$, and beam steerers ${ }^{[10,11]}$, due to their unique optical and dielectric anisotropies ${ }^{[15,16]}$, i.e., the birefringence $\Delta n=n_{e}-n_{o}$, where $n_{e}$ and $n_{o}$ are the extraordinary and ordinary refractive indices, respectively, and the dielectric anisotropy $\Delta \varepsilon=\varepsilon_{\|}-\varepsilon_{\perp}$ with $\varepsilon_{\|}$and $\varepsilon_{\perp}$ measured along and perpendicular to the optic axis, respectively. NLCs with rod-like molecules possess partial orientational order, and their average molecular orientation is called the director $\hat{\mathbf{n}}$, which is also the optic axis of the uniaxial NLCs. Traditional electro-optic devices are based on the realignment of the $\hat{\mathbf{n}}$ with the switching time on the order of milliseconds ${ }^{[15,17,18]}$. Diverse approaches have been proposed to accelerate the switching of NLCs, such as sub-millisecond electro-optic response in the bidirectional field switching mode ${ }^{[19]}$, and 0.1 ms response time by employing a dual-frequency NLC in a special geometry with a high pretilt angle ${ }^{[20]}$. The reactive monomer is also introduced into the NLCs to reduce the switching time. They are polymerized to form polymer stabilized liquid crystals (PSLCs) ${ }^{[21-25]}$, where the director of NLCs can be reoriented within $\sim 0.1 \mathrm{~ms}^{[26]}$.
Such a three-dimensional polymer structure enables the PSLC to have a fast fringe-field switching, low operating voltage ${ }^{[27]}$, low hysteresis effect, and high contrast ratio ${ }^{[28]}$.

Recently, the birefringence of NLCs with a negative dielectric anisotropy has been electrically changed on the time scale of nanoseconds for both switching-on and switching-off processes by the so-called nanosecond electrically modified order parameter (NEMOP) effect ${ }^{[29-31]}$. In the NEMOP effect, the applied electric field changes the optical property of NLCs by the enhanced uniaxial order parameters and the induced biaxial order parameters ${ }^{[29-31]}$. However, the NEMOP effect has not been explored in PSLCs.

In this work, we experimentally investigate the effect of the polymer network on the field-induced birefringence change caused by the electric modification of order parameters by varying the concentration of the reactive monomer and the polymerization condition. The field-induced birefringence change $\delta n$ is reduced by $65 \%$ via the polymerization of a mixture composed of $25 \%$ (mass fraction, hereinafter the same unless specified otherwise) reactive monomer and $75 \%$ nematic host. Both the field-on $\left(\tau_{\text {on }}\right)$ and field-off $\left(\tau_{\text {off }}\right)$ switching times are in the range of tens of nanoseconds, indicating that the polymer network does not cause a dramatic change in the response time. 


\section{Experimental Materials and Methods}

We use two commercially available NLC mixtures MJ961200 $\left(\Delta n=0.11\right.$ at $589 \mathrm{~nm}, \Delta \varepsilon=-5.6$ at $1 \mathrm{kHz}, 20^{\circ} \mathrm{C}$, purchased from Merck) and HNG7058 ( $\Delta n=0.08$ at $589 \mathrm{~nm}, \Delta \varepsilon=-9.2$ at $1 \mathrm{kHz}, 20^{\circ} \mathrm{C}$, purchased from Jiangsu Hecheng Display Technology). The host NLCs are mixed with reactive monomer 1,4-bis-[4-(3-acryloyloxypropyloxy) benzoyloxy]-2-methylbenzene (RM257, BDH, Ltd.). To induce polymerization of the reactive monomer, a small amount (about 1\%) of photo-initiator IRG651 (Aldrich) is added to the monomer-NLC mixture, which can provide free radicals when irradiated with UV light. To study the concentration dependence of the field-induced birefringence change $\delta n$, we use two PSLCs composed of MJ961200 and different mass fractions of RM257, 5\% (M5) and 15\% (M15), respectively. The mixtures M5 and M15 are polymerized at $23^{\circ} \mathrm{C}$. To explore how the polymerization temperature influences $\delta n$, we use three mixtures composed of HNG7058 and various concentrations of RM257, 10\% (HT23), 25\% (HT84), and 10\% (HT100) which are polymerized at $23^{\circ} \mathrm{C}, 84^{\circ} \mathrm{C}$, and $100^{\circ} \mathrm{C}$, respectively.

To obtain homogenous mixtures, the mixtures are dissolved in chloroform, vibrated with a vortex shaker at a speed of $1500 \mathrm{r} / \mathrm{min}$ for $10 \mathrm{~min}$, and stirred in an ultrasonic bath at $40^{\circ} \mathrm{C}$ for $75 \mathrm{~min}$. After the evaporation of chloroform in a vacuum chamber for $24 \mathrm{~h}$, the mixtures are injected into the cells in their isotropic phase. The cells are irradiated with UV light (wavelength $365 \mathrm{~nm}$, intensity $1 \mathrm{~mW} / \mathrm{cm}^{2}$ ) for $3 \mathrm{~h}$ to induce the photopolymerization.

To study the electro-optic performance, we use cells comprising two glass plates with transparent indium tin oxide (ITO) electrodes of low resistivity (between 10 and $50 \Omega /$ sq). The polyimide PI-2555 (HD MicroSystems) is coated onto the inner surfaces of two plates and then unidirectionally rubbed to provide a planar alignment of the studied NLCs and PSLCs. Two parallel glass plates are separated by silica spheres of diameter in the range of $d=2.6-6.1 \mu \mathrm{m}$. We use a He-Ne laser beam of wavelength $\lambda=632.8 \mathrm{~nm}$ to measure the electro-optic response of pure NLCs and PSLCs. The beam passes through the cell, Soleil-Babinet compensator, and two crossed polarizers. The test cells are sandwiched between two prisms so that the linearly polarized laser beam enters the PSLC slab at an angle of $45^{\circ}$, which eliminates the contribution of director fluctuations to the optical response ${ }^{[30]}$, as shown in Fig. 1(a). The director $\hat{\mathbf{n}}$ is parallel to the incident plane of the cell. The transmitted light intensity is recorded by a photodetector TIA-525 (Terahertz Technologies, response time $<1 \mathrm{~ns}$ ), which can be expressed as

$$
I(t)=I_{0} \sin ^{2}\left\{\frac{\pi\left[\delta n(t)+\Delta n_{\mathrm{eff}}\right] L}{\lambda}+\frac{\phi_{\mathrm{SB}}}{2}\right\},
$$

where $\phi_{\mathrm{SB}}$ is the variable phase retardance controlled by the Soleil-Babinet compensator, $L$ is the optical path, $I_{0}$ is the initial intensity of the laser beam, and $\Delta n_{\text {eff }}$ is the effective birefringence of the NLC in the experiment. To eliminate parasitic effects (such as light scattering), we use two compensator
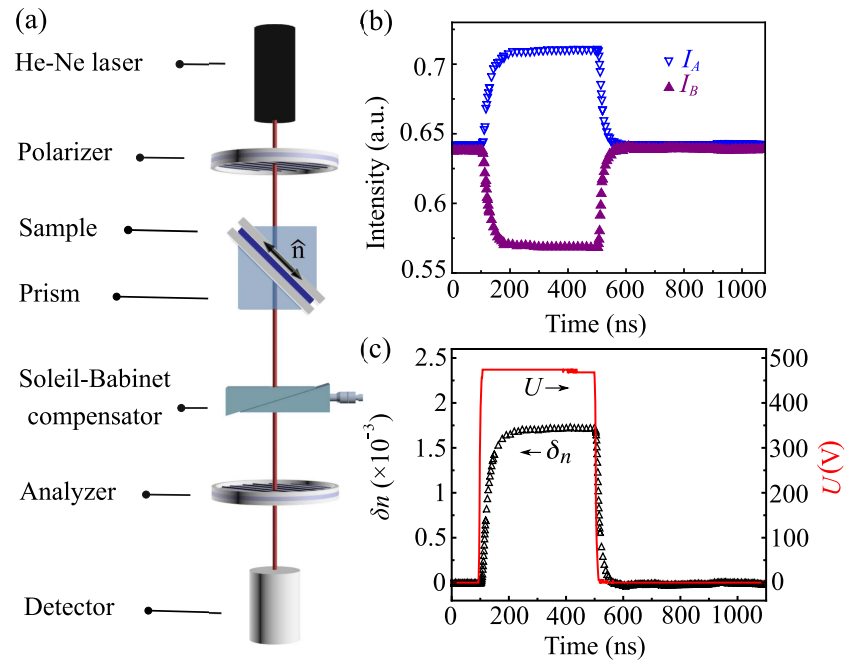

Fig. 1. (a) Experimental setup: a cell sandwiched between two right angle prisms, probed with a linearly polarized laser beam that propagates inside the nematic slab at an angle of $45^{\circ}$ with respect to the cell normal. (b) Transmitted light intensity of the cell filled with the NLC HNG7058 in response to the applied voltage pulse amplitude $U_{0}=478 \mathrm{~V}$. (c) Dynamics of the field-induced birefringence change $\delta n(t)$ of the NLC HNG7058 in response to a voltage pulse amplitude $U_{0}=478 \mathrm{~V}$. The experiments are performed at $23^{\circ} \mathrm{C}$ in a cell of thickness $d=6.1 \mu \mathrm{m}$ for the two compensator settings $\mathrm{A}$ and $\mathrm{B}$.

settings $A$ and $B$, where the transmitted light intensities are $I_{A}(t)$ and $I_{B}(t)$, respectively:

$$
\begin{aligned}
& I_{A}(t)=\sin ^{2}\left(\frac{\pi L \Delta n_{\mathrm{eff}}}{\lambda}+\frac{\phi_{A}}{2}\right)=\sin ^{2}\left(\phi_{A e}\right), \\
& I_{B}(t)=\sin ^{2}\left(\frac{\pi L \Delta n_{\mathrm{eff}}}{\lambda}+\frac{\phi_{B}}{2}\right)=\sin ^{2}\left(\phi_{B e}\right) .
\end{aligned}
$$

We choose two compensator settings, which satisfy $\phi_{B e}=\phi_{A e}+\frac{\pi}{2}$; one can obtain

$$
\Delta I_{A}(t)-\Delta I_{B}(t)=\cos \left(2 \phi_{A e}\right)-\cos \left[2\left(\delta \phi+\phi_{A e}\right)\right],
$$

where $\Delta I_{A}=I_{A}(t)-I_{A}(0)$ and $\Delta I_{B}=I_{B}(t)-I_{B}(0)$.

The laser beam propagates inside the nematic slab at an angle of $45^{\circ}$ with respect to the cell normal, $L=\sqrt{2} d$. Considering $k=\frac{2 \pi}{\lambda}$, the field-induced birefringence change can be expressed as

$$
\delta n=\frac{-\arccos \left(1-\Delta I_{A}-\Delta I_{B}\right)-2 \arccos \left[1-2 I_{A}(t)\right]}{\sqrt{2} k d} .
$$

The dynamics of the transmitted light intensity of a cell filled with NLC HNG7058 is obtained with the settings $I_{A}(0)=$ $I_{B}(0), \phi_{A}=0.35 \pi$, and $\phi_{B}=0.85 \pi$, as shown in Fig. 1(b). $I_{A}(0)$ and $I_{B}(0)$ are the transmitted light intensity measured at the field-free initial state. Figure 1(c) shows the dynamics of the field-induced birefringence change of the NLC HNG7058 in 
response to the voltage pulse amplitude $U_{0}=478 \mathrm{~V}$, which is calculated from the data in Fig. 1(b) by using Eq. (5).

\section{Experimental Results}

\subsection{Effect of monomer concentration on the field-induced birefringence change}

We investigate the field-induced birefringence change $\delta n$ and the switching-on and switching-off times in the pure NLC MJ961200 and the PSLCs M5 and M15 before and after polymerization. The maximum field-induced birefringence change $\delta n_{\max }$ of the mixtures decreases as the concentration of reactive monomer RM257 in the nematic MJ961200 increases from 0\% to $5 \%$ and then 15\%, as shown in Figs. 2(a), 2(b), and 3(a), 3(b). The reduction in $\delta n_{\max }$ measured after polymerization is larger than that obtained before polymerization, as shown in Figs. 2 and 3. Compared with MJ961200, $\delta n_{\max }$ of M5 and M15 decreases slightly before polymerizing, as shown in Figs. 2(a), 2 (b) and 3(a), 3(b). However, after polymerizing, $\delta n_{\max }$ of M5 and M15 decreases by $9 \%$ and $30 \%$, respectively, as shown in Figs. 2(a), 2(b), and 3(a), 3(b). There is an obvious contrast between the reduction of $\delta n_{\max }$ for M5 and M15. The latter is nearly $21 \%$ lower than the former, which demonstrates that with a higher doping concentration of reactive monomer the decrease in $\delta n_{\max }$ is larger. The relationship between the electric-field and $\delta n_{\max }$ is generally quadratic, with a few deviations observed at high electric field intensity, as shown in Figs. 2(b) and 3(b).
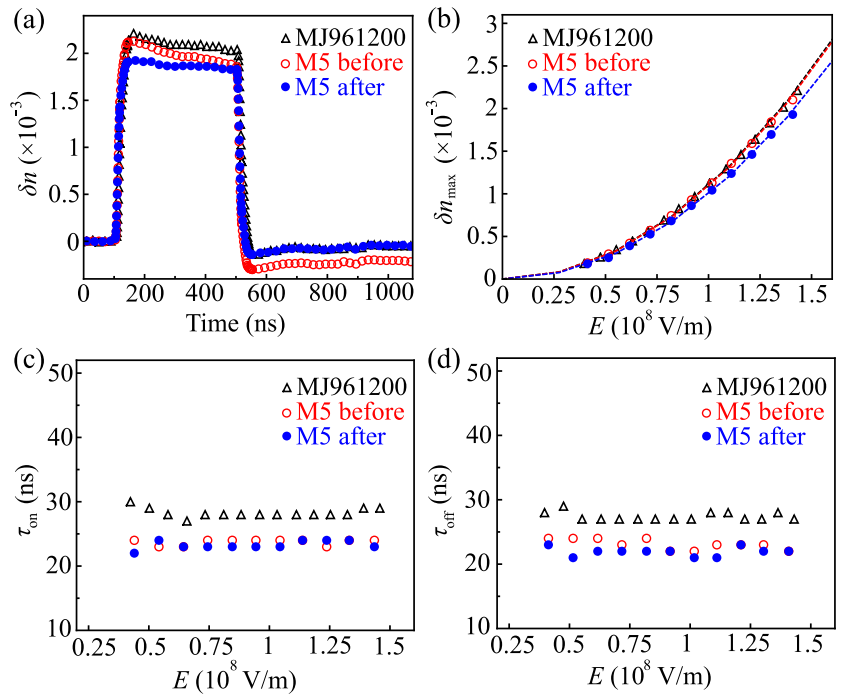

Fig. 2. Electro-optic responses of the NLC mixtures MJ961200 and M5 before and after polymerization. (a) Dynamics of field-induced birefringence change $\delta n(t)$ in response to an electric field of amplitude $E=1.44 \times 10^{8} \mathrm{~V} / \mathrm{m}$. Dependences of (b) the maximum field-induced birefringence change $\delta n_{\max }$ (c) the switching-on time $\tau_{\text {on }}$ and (d) the switching-off time $\tau_{\text {off }}$ on the applied electric fields. The cell thicknesses of the MJ961200 cell and the M5 cell are $6.1 \mu \mathrm{m}$ and $4.6 \mu \mathrm{m}$, respectively. The working temperature is $T=21.5^{\circ} \mathrm{C}$.
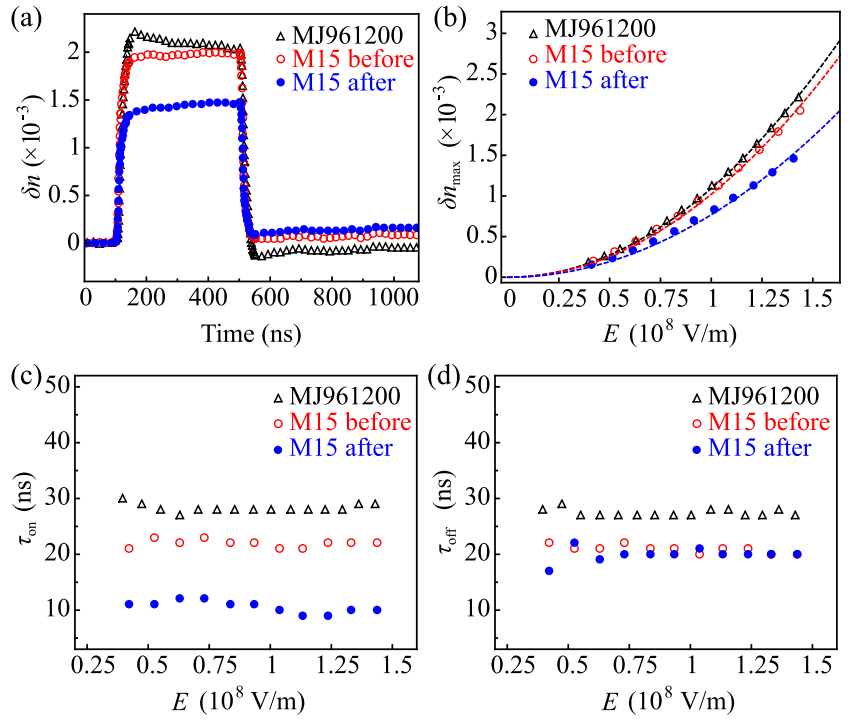

Fig. 3. Electro-optic responses of the NLC mixtures MJ961200 and M15 before and after polymerization. (a) Dynamics of field-induced birefringence $\delta n(t)$ in response to an electric field of amplitude $E=1.44 \times 10^{8} \mathrm{~V} / \mathrm{m}$. Dependences of (b) the maximum field-induced birefringence change $\delta n_{\text {max }}$ (c) the switching-on time $\tau_{\text {on, }}$ and (d) the switching-off time $\tau_{\text {off }}$ on the applied electric fields. The cell thicknesses of the MJ961200 cell and the M15 cell are $6.1 \mu \mathrm{m}$ and $4.6 \mu \mathrm{m}$, respectively. The working temperature is $T=21.5^{\circ} \mathrm{C}$.

From the view of practical applications, we use the industrystandard 10\%-90\% switching time to characterize the electrooptic response time of the mixtures. Namely, the switching-on $\left(\tau_{\text {on }}\right)$ and switching-off $\left(\tau_{\text {off }}\right)$ times of the mixtures are calculated as the $\delta n$ changes from $10 \%-90 \%$ of the $\delta n_{\max 1}-\delta n_{\min 1}$ and from $90 \%-10 \%$ of the $\delta n_{\max 2}-\delta n_{\min 2}$, respectively. The $\delta n_{\max 1}-\delta n_{\min 1}$ and $\delta n_{\max 2}-\delta n_{\min 2}$ are the differences between the maximum and minimum values of the switchingon $\left(\tau_{\text {on }}\right)$ and switching-off $\left(\tau_{\text {off }}\right)$ processes, respectively.

Polymerization of RM257 speeds up the switching-on and switching-off processes of the MJ961200 mixtures, as shown in Figs. 2(c), 2(d), and 3(c), 3(d). Both average switching times $\bar{\tau}_{\text {on }}$ and $\bar{\tau}_{\text {off }}$ of the mixture M5 ( 20-25 ns) are several nanoseconds faster than those of MJ961200, as shown in Figs. 2(c) and 2(d). The response of the PSLC is faster with the increase of concentration of RM257 in the mixture. For example, the average switching-on time $\bar{\tau}_{\text {on }}$ of the polymerized M15 is $\sim 10 \mathrm{~ns}$, which is half of that for MJ961200 and even less for M5. The average switching-off time $\bar{\tau}_{\text {off }}$ of the M15 after polymerizing is about $20 \mathrm{~ns}$, which is $10 \mathrm{~ns}$ faster than that of MJ961200, as shown in Figs. 3(c) and 3(d).

\subsection{Effect of polymerization temperature on the field- induced birefringence change}

We further investigate $\delta n_{\max }, \tau_{\text {on }}$, and $\tau_{\text {off }}$ in the pure NLC HNG7058 and the PSLCs HT23 and HT100 before and after polymerization. $\delta n_{\max }$ of the mixtures decreases with the polymerization temperature increasing to $23^{\circ} \mathrm{C}$ and $100^{\circ} \mathrm{C}$, as shown in 

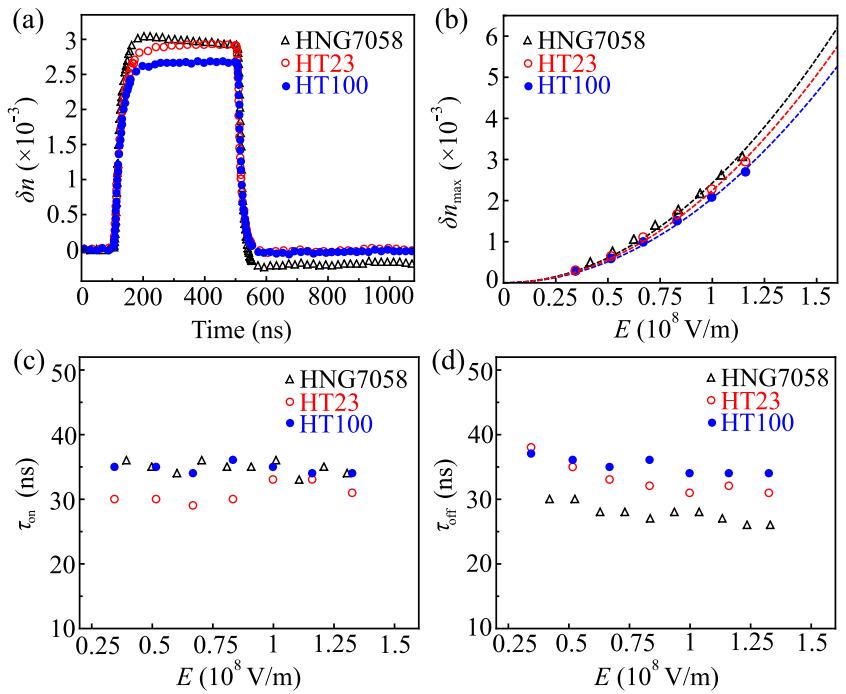

Fig. 4. Electro-optic responses of the NLC mixtures HNG7058, HT23, and HT100. (a) Dynamics of field-induced birefringence change $\delta n(t)$ in response to an electric field of amplitude $E=1.44 \times 10^{8} \mathrm{~V} / \mathrm{m}$. Dependences of (b) the $\delta n_{\text {max }}$ (c) the switching-on time $\tau_{\text {on, }}$ and (d) the switching-off time $\tau_{\text {off }}$ on the applied electric fields. The cell thicknesses of the HNG7058 cell, HT23 cell, and HT100 cell are $4.6 \mu \mathrm{m}, 2.9 \mu \mathrm{m}$, and $2.9 \mu \mathrm{m}$, respectively. The working temperature is $T=21.5^{\circ} \mathrm{C}$

Figs. 4(a) and 4(b). Compared to the non-doped NLC HNG7058, $\delta n_{\max }$ of HT23 changes slightly, and $\delta n_{\max }$ of HT100 decreases by $12 \%$, as shown in Figs. 4(a) and 4(b). The average switching-on time $\bar{\tau}_{\text {on }}$ of HT23 is about 5 ns shorter than that of HNG7058, and the average switching-off time $\bar{\tau}_{\text {off }}$ of HT23 and
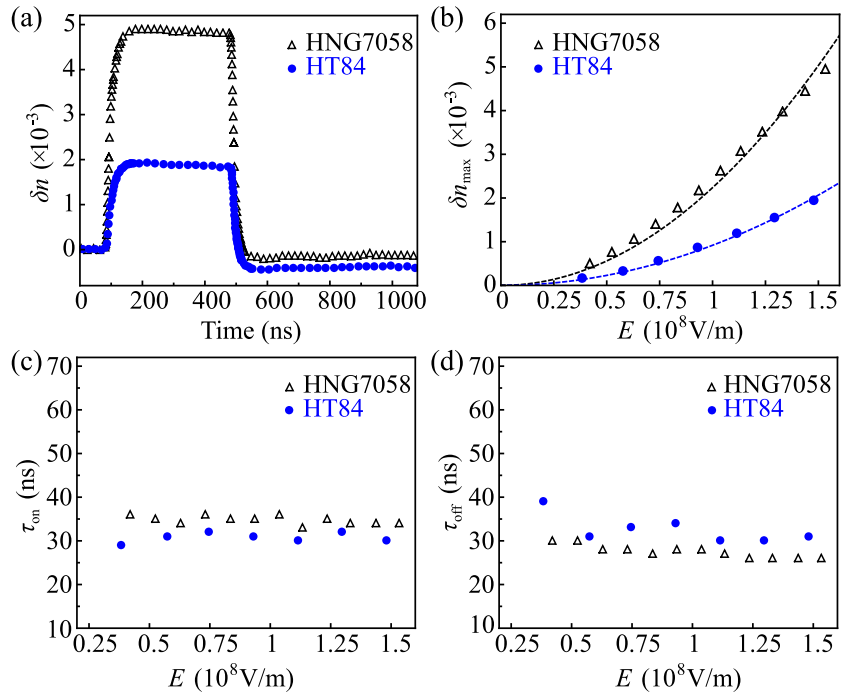

Fig. 5. Electro-optic responses of the NLC mixtures HNG7058 and HT84. (a) Dynamics of field-induced birefringence change $\delta n(t)$ in response to an electric field of amplitude $E=1.44 \times 10^{8} \mathrm{~V} / \mathrm{m}$. Dependences of (b) the $\delta n_{\text {max }}$ (c) the switching-on time $\tau_{\text {on, }}$ and (d) the switching-off time $\tau_{\text {off }}$ on the applied electric fields. The cell thicknesses of the HNG7058 cell and the HT84 cell are $4.6 \mu \mathrm{m}$ and $2.6 \mu \mathrm{m}$, respectively. The working temperature is $T=21.5^{\circ} \mathrm{C}$.
HT100 is about $10 \mathrm{~ns}$ longer than that of HNG7058. The average response times $\bar{\tau}_{\text {on }}$ and $\bar{\tau}_{\text {off }}$ of HT100 are several nanoseconds longer than those of HT23, as shown in Figs. 4(c) and 4(d).

Taking advantage of a higher concentration of RM257 and a high polymerization temperature, we prepare another mixture composed of HNG7058 doped with $25 \%$ reactive monomer RM257 and then investigate its $\delta n_{\max }, \tau_{\text {on }}$, and $\tau_{\text {off }}$. The mixture is polymerized at $84^{\circ} \mathrm{C}$, which is $1.5^{\circ} \mathrm{C}$ below the nematic-toisotropic phase transition temperature $T_{\mathrm{NI}}$. The $\delta n_{\max }$ of HT84 is about $35 \%$ of the $\delta n_{\max }$ of HNG7058, as shown in Figs. 5(a) and 5(b). The $65 \%$ decrease of $\delta n_{\max }$ in HT84 is larger than that of the other PSLCs in this work. Compared to HNG7058, the PSLC HT84 has a smaller $\tau_{\text {on }}$ and larger $\tau_{\text {off }}$. Both of them are on the order of tens of nanoseconds, as shown in Figs. 5(c) and $5(\mathrm{~d})$.

\section{Discussions and Conclusions}

We demonstrate that the electro-optic response coming from the nanosecond electrically modified order parameter effect can be attenuated in PSLCs. The results show that the characteristic parameters $\delta n_{\max }, \tau_{\text {on }}$, and $\tau_{\text {off }}$ of the optic response can be tuned by varying the doping concentration of RM257 in host NLCs and curing the active monomer at different temperatures. The reduction effect of the optic response is stronger when a higher concentration active monomer is doped. The $30 \%$ reduction of $\delta n_{\max }$ in the NLC MJ961200 is obtained from the PSLC doped with 15\% RM257. The comparison of the electro-optical responses between HT23 and HT84 reveals that a greater reduction in $\delta n_{\text {max }}$ occurs in the PSLC, which is polymerized at the high temperature close to $T_{\mathrm{NI}}$. The value of $\delta n_{\max }$ can be dramatically reduced by $65 \%$. The response time of the studied PSLCs is in the range of $10-40$ ns. The strong interaction between polymer rods and NLC molecules at the polymer/ NLC interface stabilizes the nematic order close to the polymer, which could be the potential mechanism of the reduction of birefringence change caused by doping more reactive monomers. When polymerization occurs at high temperature, the number of polymer rods may increase and cause an increase in the area of the polymer surfaces, affecting more NLC molecules. Thus, the order parameter of NLCs is further enhanced. To obtain a concrete and comprehensive understanding of the mechanism of the reduction effect, systematic studies need to be performed using more sophisticated characterization techniques in the future. The reduction of the field-induced birefringence change by PSLC could be used to protect some liquid-crystal-based precise devices exposed in the surroundings where an unexpected electric field occurs and causes damage to the devices.

\section{Acknowledgement}

This work was supported by the National Natural Science Foundation of China (No. 52003115) and Natural Science Foundation of Jiangsu Province (Nos. BK20212004 and BK20200320). The authors gratefully appreciate Prof. O. D. Lavrentovich, 
Dr. S. V. Shiyanovskii, and Dr. V. Borshch for their constructive discussions.

${ }^{\dagger}$ These authors contributed equally to this work.

\section{References}

1. Y. Q. Lu and Y. Li, "Planar liquid crystal polarization optics for near-eye displays," Light Sci. Appl. 10, 122 (2021).

2. S. R. Seyednejad and M. R. Mozaffari, "Conically degenerate anchoring effect in planar nematic-liquid-crystal shells," Phys. Rev. E 104, 014701 (2021).

3. Y. Jiang, G. Qin, X. Xu, L. Zhou, S. Lee, and D. K. Yang, "Image flickering-free polymer stabilized fringe field switching liquid crystal display," Opt. Express 26, 32640 (2018).

4. R. Kumar and K. K. Raina, "Electrically modulated fluorescence in optically active polymer stabilised cholesteric liquid crystal shutter," Liq. Cryst. 41, 228 (2014).

5. H. Ren, S. Xu, D. Ren, and S. T. Wu, "Novel optical switch with a reconfigurable dielectric liquid droplet," Opt. Express 19, 1985 (2011).

6. J. Sun, Y. Chen, and S. T. Wu, "Submillisecond-response and scattering-free infrared liquid crystal phase modulators," Opt. Express 20, 20124 (2012).

7. Y. H. Lin, H. Ren, Y. H. Wu, Y. Zhao, J. Fang, Z. Ge, and S. T. Wu, "Polarization-independent liquid crystal phase modulator using a thin polymer-separated double-layered structure," Opt. Express 13, 8746 (2005).

8. B. Wei, Y. Zhang, P. Li, S. Liu, W. Hu, Y. Lu, Y. Wu, X. Dou, and J. Zhao, "Liquid-crystal splitter for generating and separating autofocusing and autodefocusing circular Airy beams," Opt. Express 28, 26151 (2020).

9. S. An, Y. Shi, Z. Yi, C. Liu, T. Sun, J. Lv, L. Yang, and P. Chu, "Ultra-short dual-core GaAs photonic crystal fiber splitter filled with nematic liquid crystal," Opt. Eng. 60, 056104 (2021).

10. S. Ge, P. Chen, Z. Shen, W. Sun, X. Wang, W. Hu, Y. Zhang, and Y. Lu, "Terahertz vortex beam generator based on a photopatterned large birefringence liquid crystal," Opt. Express 25, 12349 (2017).

11. Z. He, K. Yin, and S. T. Wu, "Miniature planar telescopes for efficient, wideangle, high-precision beam steering," Light Sci. Appl. 10, 134 (2021).

12. Z. X. Li, Y. P. Ruan, P. Chen, J. Tang, W. Hu, K. Y. Xia, and Y. Q. Lu, "Liquid crystal devices for vector vortex beams manipulation and quantum information applications," Chin. Opt. Lett. 19, 112601 (2021).

13. J. Yan, X. Fan, Y. Liu, Y. Yu, Y. Fang, and R. Z. Li, "Passive patterned polymer dispersed liquid crystal transparent display," Chin. Opt. Lett. 20, 013301 (2022).

14. Y. Zhang, B. Wei, S. Liu, P. Li, X. Chen, Y. Wu, X. Dou, and J. Zhao, "Circular Airy beams realized via the photopatterning of liquid crystals," Chin. Opt. Lett. 18, 080008 (2020).

15. D. K. Yang and S. T. Wu, Fundamentals of Liquid Crystal Devices (Wiley, 2014).

16. B. X. Li, V. Borshch, R. L. Xiao, S. Paladugu, T. Turiv, S. V. Shiyanovskii, and O. D. Lavrentovich, "Electrically driven three-dimensional solitary waves as director bullets in nematic liquid crystals," Nat. Commun. 9, 2912 (2018).
17. I. C. Khoo and S. L. Zhuang, "Nonlinear optical amplification in a nematic liquid crystal above the Frederiks transition," Appl. Phys. Lett. 37, 3 (1980).

18. I. C. Khoo, Y. Liang, and H. Li, "Observation of stimulated orientational scattering and cross-polarized self-starting phase conjugation in a nematic liquid-crystal film," Opt. Lett. 20, 130 (1995).

19. A. R. Geivandov, M. I. Barnik, V. S. Palto, I. V. Simdyankin, and S. P. Palto, "Submillisecond electro-optical response of a nematic liquid crystal in the bidirectional field switching mode," Crystallogr. Rep. 63, 971 (2018).

20. A. B. Golovin, S. V. Shiyanovskii, and O. D. Lavrentovich, "Fast switching dual-frequency liquid crystal optical retarder, driven by an amplitude and frequency modulated voltage," Appl. Phys. Lett. 83, 3864 (2003).

21. S. Guo, X. Liang, H. Zhang, W. Shen, C. Li, X. Wang, C. Zhang, L. Zhang, and H. Yang, "An electrically light-transmittance-controllable film with a lowdriving voltage from a coexistent system of polymer-dispersed and polymer-stabilised cholesteric liquid crystals," Liq. Cryst. 45, 1854 (2018).

22. C. Y. Li, X. Wang, X. Liang, J. Sun, C. X. Li, S. F. Zhang, L. Y. Zhang, H. Q. Zhang, and H. Yang, "Electro-optical properties of a polymer dispersed and stabilized cholesteric liquid crystals system constructed by a stepwise UV-initiated radical/cationic polymerization," Crystals 9, 282 (2019).

23. G. Pan, H. Cao, R. Guo, W. Li, J. Guo, Z. Yang, W. Huang, W. He, X. Liang, D. Zhang, and H. Yang, "A polymer stabilized liquid crystal film with thermal switching characteristics between light transmission and adjustable light scattering," Opt. Mater. 31, 1163 (2009).

24. L. Zhang, K. Li, W. Hu, H. Cao, Z. Cheng, W. He, J. Xiao, and H. Yang, "Broadband reflection mechanism of polymer stabilised cholesteric liquid crystal (PSChLC) with pitch gradient," Liq. Cryst. 38, 673 (2011).

25. J. Guo, H. Cao, J. Wei, D. Zhang, F. Liu, G. Pan, D. Zhao, W. He, and H. Yang, "Polymer stabilized liquid crystal films reflecting both right- and leftcircularly polarized light," Appl. Phys. Lett. 93, 201901 (2008).

26. X. Li, X. Du, P. Guo, J. Zhu, W. Ye, Q. Xu, and Y. Sun, "Fast switchable dualmodel grating by using polymer-stabilized sphere phase liquid crystal," Polymers 10, 884 (2018).

27. R. Zhao, X. Li, K. Wang, H. Huai, H. Ma, and Y. Sun, "Effect of the introduction of mono-functional monomer on the electro-optic properties of reverse-mode polymer stabilised cholesteric liquid crystal," Liq. Cryst. 48, $1162(2021)$.

28. L. Weng, A. Varanytsia, K. H. Chang, and L. C. Chien, "Simulation and fabrication of a fast fringe-field switching liquid crystal with enhanced surface anchoring enabled by controlled polymer topology," J. Soc. Inf. Display. 24, 621 (2016).

29. V. Borshch, S. V. Shiyanovskii, and O. D. Lavrentovich, "Nanosecond electro-optic switching of a liquid crystal," Phys. Rev. Lett. 111, 107802 (2013).

30. V. Borshch, S. V. Shiyanovskii, B. X. Li, and O. D. Lavrentovich, "Nanosecond electro-optics of a nematic liquid crystal with negative dielectric anisotropy," Phys. Rev. E 90, 062504 (2014).

31. B. X. Li, S. V. Shiyanovskii, and O. D. Lavrentovich, "Nanosecond switching of micrometer optical retardance by an electrically controlled nematic liquid crystal cell," Opt. Express 24, 29477 (2016). 